\title{
Could cadmium be responsible for some of the neurological signs and symptoms of Myalgic Encephalomyelitis/Chronic Fatigue Syndrome
}

\author{
Stefania Pacini ${ }^{\text {a,* }}$, Maria G. Fiore ${ }^{\mathrm{b}}$, Stefano Magherini ${ }^{\mathrm{a}}$, Gabriele Morucci ${ }^{\mathrm{a}}$, Jacopo J.V. Branca ${ }^{\text {, }}$ \\ Massimo Gulisano ${ }^{\mathrm{a}}$, Marco Ruggiero ${ }^{\mathrm{b}}$ \\ ${ }^{a}$ Department of Anatomy, Histology and Forensic Medicine, University of Firenze, Viale Morgagni 85, 50134 Firenze, Italy \\ ${ }^{\mathrm{b}}$ Department of Experimental Pathology and Oncology, University of Firenze, Viale Morgagni 50, 50134 Firenze, Italy
}

\section{A R T I C L E I N F O}

Article history:

Received 24 May 2012

Accepted 17 June 2012

\begin{abstract}
A B S T R A C T
According to the World Health Organization, Myalgic Encephalomyelitis/Chronic Fatigue Syndrome (ME/ CFS) is a neurological disease characterized by widespread inflammation and multi-systemic neuropathology. Aetiology and pathogenesis are unknown, and several agents have been proposed as causative agents or as factors perpetuating the syndrome. Exposure to heavy metals, with particular reference to mercury and gold in dental amalgams, has been considered among the triggers of ME/CFS. Here we hypothesize that cadmium, a widespread occupational and environmental heavy metal pollutant, might be associated with some of the neurological findings described in ME/CFS. In fact, ME/CFS patients show a decrease of the volume of the gray matter in turn associated with objective reduction of physical activity. Cadmium induces neuronal death in cortical neurons through a combined mechanism of apoptosis and necrosis and it could then be hypothesized that cadmium-induced neuronal cell death is responsible for some of the effects of cadmium on the central nervous system, i.e. a decrease in attention level and memory in exposed humans as well as to a diminished ability for training and learning in rats, that are symptoms typical of ME/CFS. This hypothesis can be tested by measuring cadmium exposure in a cohort of ME/CFS patients compared with matched healthy controls, and by measuring gray matter volume in un-exposed healthy controls, exposed non-ME/CFS subjects, un-exposed ME/CFS patients and exposed ME/CFS patients. In addition, we hypothesize that cadmium exposure could be associated with reduced cerebral blood flow in ME/CFS patients because of the disruptive effects of cadmium on angiogenesis. In fact, cadmium inhibits angiogenesis and low global cerebral flow is associated with abnormal brain neuroimaging results and brain dysfunction in the form of reduced cognitive testing scores in ME/CFS patients. This hypothesis can be tested by measuring cerebral cortex blood flow in un-exposed healthy controls, exposed non-ME/CFS subjects, un-exposed ME/CFS patients and exposed ME/CFS patients. If our hypothesis is demonstrated correct, the consequences could affect prevention, early diagnosis, and treatment of ME/CFS. Implications in early diagnosis could entail the evaluation of symptoms typical of ME/CFS in cadmium-exposed subjects as well as the search for signs of exposure to cadmium in subjects diagnosed with ME/CFS. Nutritional supplementation of magnesium and zinc could then be considered, since these elements have been proposed in the prophylaxis and therapy of cadmium exposure, and magnesium was demonstrated effective on ME/CFS patients' symptom profiles.
\end{abstract}

(c) 2012 Elsevier Ltd. All rights reserved.

\section{Introduction}

Myalgic Encephalomyelitis/Chronic Fatigue Syndrome (ME/CFS) is the term used to define a complex array of symptoms that includes, but is not limited to, profound, debilitating fatigue, lasting for at least six months and resulting in a substantial reduction of daily activities [1]. It is classified as a neurological disease in the World Health Organization's International Classification of Diseases

\footnotetext{
* Corresponding author. Tel.: +39 055 4271810; fax: +39 0554379500 .

E-mail address: stefania.pacini@unifi.it (S. Pacini).
}

(ICD G93.3), and it is characterized by widespread inflammation and multi-systemic neuropathology [2]. Although according to the recent International Consensus Criteria it is probably more appropriate to use only the term "Myalgic Encephalomyelitis" [2], in this article we refer to the syndrome using the traditional double acronym ME/CFS. Under this definition, it is estimated that the syndrome affects millions people worldwide, and its prevalence is calculated in between 400 and 2500 adults per 100,000 population [3].

Both aetiology and pathogenesis are unknown, and a number of agents have been proposed either as causative agents or as factors contributing to the perpetuation of the symptoms. As in many 
syndrome, it is likely that there are multiple causes leading to a common clinical picture. Although a trigger cannot be necessarily considered an etiological agent, according to a recent review, several events may act as triggers, from external environmental or infectious events, such as chemical exposure or infections, to psychological and social factors that may be critical in maintaining the chronic condition of the syndrome [4]. The hypothesis of a retrovirus being associated with ME/CFS was postulated in 2009 [5], but further studies rejected this hypothesis [6], and, as of today, the causes of ME/CFS remain obscure. Drawing an analogy from the evolution of the human brain, ME/CFS may even be regarded as a "phylogenic disease" [7-11], according to principle of "integrated phylogeny" of the primate brain [12].

Exposure to heavy metals, with particular reference to dental amalgams, has been considered among the triggers of ME/CFS, and the attention has been focused mostly on mercury and gold $[13,14]$. According to Stejskal et al. [13], heavy metals might be responsible for inflammatory processes that may modulate the hypothalamic-pituitary-adrenal axis and trigger the multiple, non-specific, symptoms characterizing ME/CFS. These Authors noticed in 1999 that in certain ME/CFS patients cadmium was a sensitizer for blood lymphocytes, and this observation was confirmed in 2006 by Valentine-Thon et al. [15] who described cadmium-sensitization in metal-exposed individuals showing a number of symptoms superimposable to those of ME/CFS. Nevertheless, the precise role of cadmium in the aetiology or pathogenesis of $\mathrm{ME} /$ CFS is not well understood, and the target organ of cadmium toxicity responsible for ME/CFS symptoms is not known. Probably, also because of this lack of knowledge, ME/CFS is not considered among the risk hazards associated with cadmium exposure. Here we hypothesize that cadmium, one of the most important occupational and environmental heavy metal pollutants, might be associated with, or responsible for, some of the neurological findings recently described in $\mathrm{ME} / \mathrm{CFS}$.

\section{The hypothesis}

Cadmium might cause brain alterations triggering or perpetuating the symptoms of ME/CFS. The hypothesis stems from the observation that the effects of cadmium on neurons and blood vessels are consistent with the pathologic alterations observed in ME/ CFS patients.

\section{Cadmium and neuronal cell death in ME/CFS}

Since ME/CFS is considered a neurological disorder, several attempts have been made to detect alterations in the brain of ME/ CFS patients. A recent study using a fully automated, observerindependent procedure to study morphological alterations of the central nervous system, demonstrated that ME/CFS patients had an average decrease of the volume of the gray matter of about $8 \%$ compared to matched healthy controls [16]. The gray matter volume reduction was significantly associated with objective reduction of physical activity in ME/CFS patients [16]. Gray matter volume reduction was not localized in any particular area of the brain and it was not associated with a concomitant reduction in white matter, thus indicating that the phenomenon was specific to neurons. Gray matter volume reduction could then be held accountable for most of the symptoms of ME/CFS. It is therefore interesting to notice that it was recently demonstrated that cadmium induced neuronal death in cortical neurons through a combined mechanism of apoptosis and necrosis involving reactive oxygen species generation and lipid peroxidation [17]. It could then be hypothesized that cadmium-induced neuronal cell death might be responsible for some of the well known effects of cadmium on the central nervous system, with particular reference to a decrease in attention level and memory in exposed humans [18] as well as to a diminished ability for training and learning in rats exposed to cadmium during their neonatal life [19]. It is worth mentioning, in the context of our hypothesis, that attention and memory deficit are among the most debilitating symptoms in ME/CFS patients [20,21]. Although the effects of cadmium on neuronal cells in culture have been described in several papers [22], its effects on human central nervous system neurons in vivo have not yet been demonstrated. However, the recently described cadmium-induced apoptosis of the motor neurons of the ventral horns in cultured explants from human foetal spinal cords (10-11 weeks gestational age) [23], suggests that the effects observed in vitro could actually occur also in vivo. This notwithstanding, the demonstration of a link between cadmium exposure and neuronal cell death in living adult human brain is missing as well as still missing is the demonstration of a link between cadmium exposure and ME/ CFS.

In order to test the hypothesis that cadmium exposure is associated with ME/CFS, we propose to measure cadmium exposure in a cohort of ME/CFS patients compared with matched healthy controls. Cadmium exposure could be assessed by different methods; determination of hair mineral contents by inductively coupled plasma-mass spectrometry could be a simple and non-expensive method [24]. This method allows quick determination of absolute cadmium concentration as well as measurement of the ratios between physiological minerals and cadmium. For example, in a certified ME/CFS patient, we observed that hair cadmium content was within the normal range, whereas the ratios calcium/cadmium, magnesium/cadmium and sodium/cadmium, were well below the normal ranges. This finding, although anecdotic and presented here only to support the feasibility of testing our hypothesis, might suggest that cadmium could substitute for those physiological minerals at the cellular level.

The hypothesis that cadmium exposure is associated with neuron reduction in vivo and with ME/CFS, could be tested by measuring gray matter volume in un-exposed healthy controls, exposed non-ME/CFS subjects, un-exposed ME/CFS patients and exposed $\mathrm{ME} / \mathrm{CFS}$ patients. This latter approach, however, would require an imaging procedure that is simpler and less expensive than that described in [16] which used high resolution structural magnetic resonance imaging. To this end, we developed a modified procedure for transcranial sonography using a linear probe that is routinely used for muscle-skeletal ultrasound imaging instead of the sector array with a small footprint used for traditional transcranial sonography. We observed that such a procedure allowed detailed visualization of the cortex of the temporal lobe, a finding that is in itself potentially important for the diagnosis and follow-up of ME/CFS patients. Thus, it is well known that the temporal lobe is involved in the processing of those functions, such as semantics and memory, that are often compromised in ME/CFS patients [25]. The parameters adopted for visualization of the temporal cortex allowed to distinguish the meninges, the subarachnoidal space and the cortex (Fig. 1). The meninges appeared as a well organized array of layers of about $4 \mathrm{~mm}$ thickness. The thickness of the cortex measured in our study $(3.9 \mathrm{~mm})$ led us to hypothesize that we were observing the temporal areas designated by von Economo as TG and TE, i.e. those areas involved in the control of eye movements and balance in standing position (area TE), social behaviour, mood and decision making (area TG) [26]. It is worth remembering that most of these functions are altered to various degree in ME/ CFS patients $[2,4]$. As a matter of fact, many of the neurocognitive deficits used to define the syndrome (difficulty in processing information, slowed thought, impaired concentration, disorientation, cognitive overload, difficulty with making decisions, slowed speech, acquired or exertional dyslexia, short-term memory loss, 


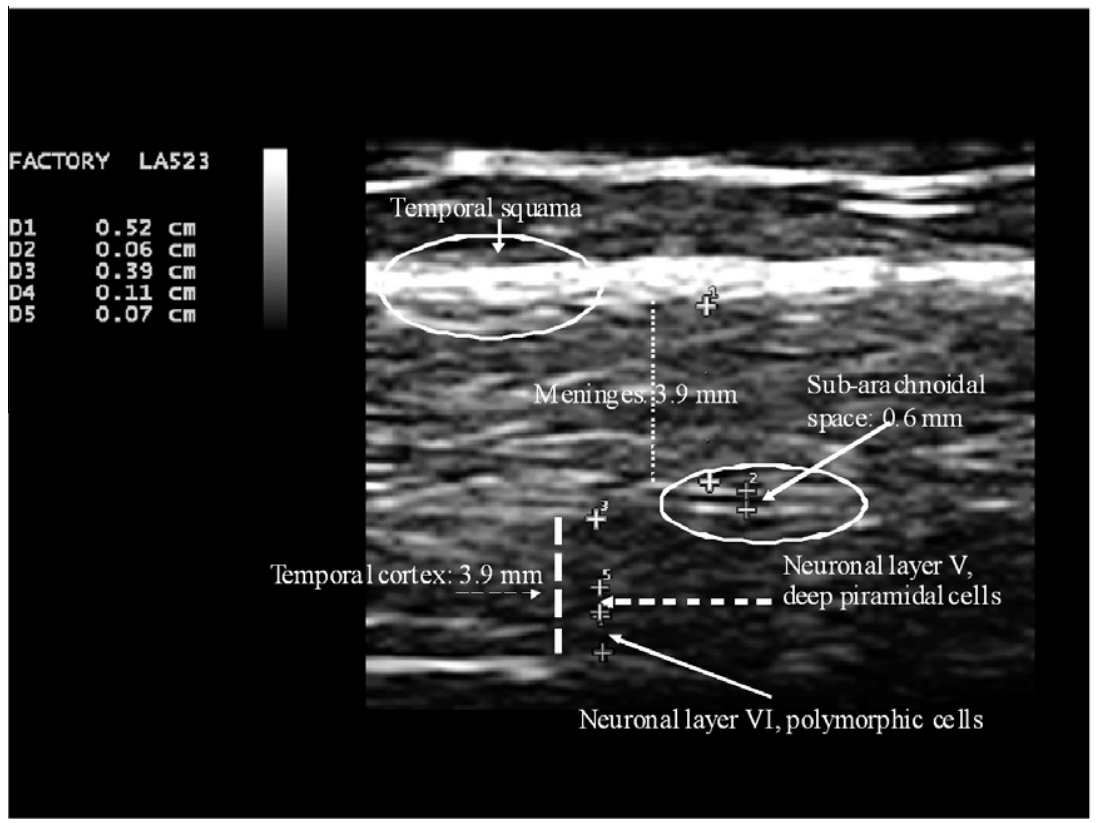

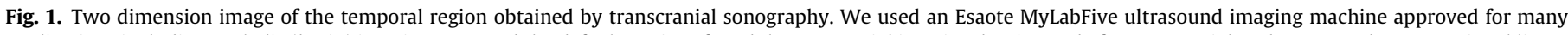

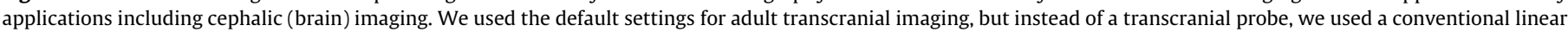

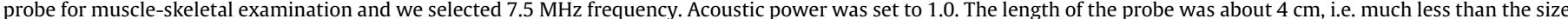

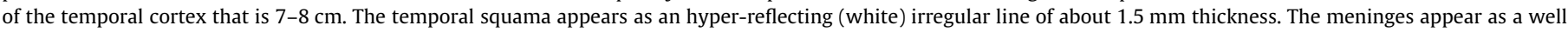

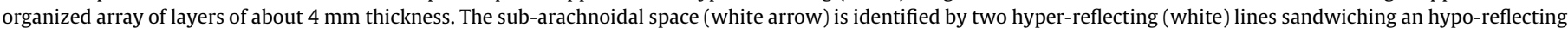

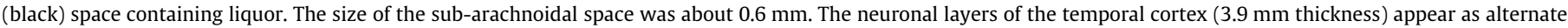

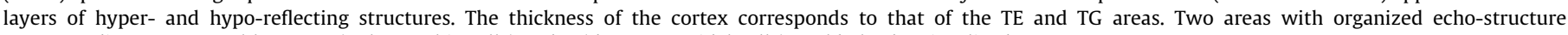
corresponding to neuronal layers VI (polymorphic cells) and V (deep pyramidal cells) could also be visualized.

poor working memory) [2] seem to pertain to functions controlled by the temporal lobe.

The spatial resolution and the ability to visualize structures of 200-300 $\mu \mathrm{m}$ size, led us to hypothesize that the linear structures (alternate gray-white stripes) parallel to the sub-arachnoidal space could be referred to the well known neuronal layers of the cortex [27]. Detailed study of these layers in ME/CFS subjects exposed or non-exposed to cadmium might prove instrumental in demonstrating whether cadmium affects neuronal viability in vivo and/ or whether these changes are related with ME/CFS.

\section{Cadmium and angiogenesis in ME/CFS}

We recently described the paradoxical effects of cadmium on the angiogenesis induced by cancer cells [28]. The effect was termed "paradoxical" because cadmium, a known carcinogen, exerted an apparent anti-cancer effect by inhibiting cancer cell-stimulated angiogenesis. The effects of cadmium on angiogenesis, however, are complex and a dual effect has been recently described: it appears that at low dose it stimulates angiogenesis, whereas at higher doses it inhibits new blood vessel formation [29]. The role

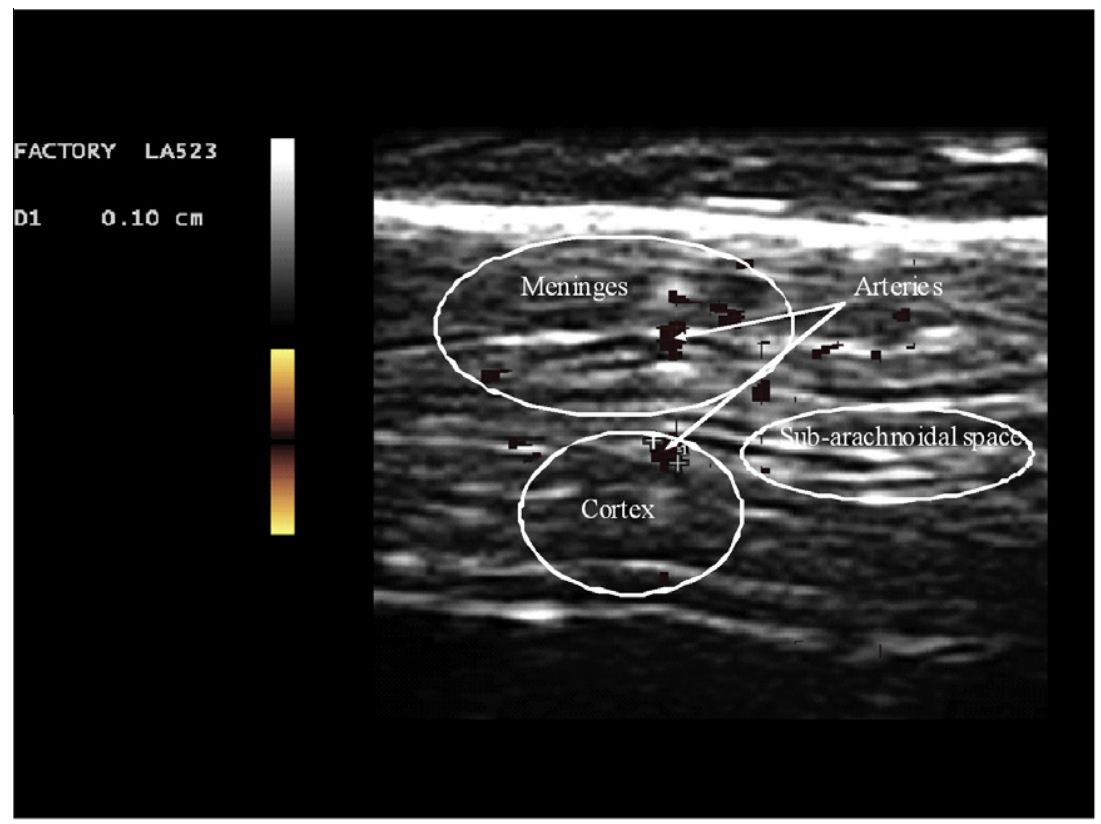

Fig. 2. Pulsating arterial blood vessels in the meninges and in the temporal cortex just below the sub-arachnoidal space. 
of angiogenesis in ME/CFS has received little or no attention so far. Cerebral blood flow is decreased in most, but not all, ME/CFS patients [30], and the cortical blood flow appears to be most affected [31]. The reduction of cerebral blood flow led the Authors to hypothesize that low global cerebral flow was associated with abnormal brain neuroimaging results and objective evidence of brain dysfunction in the form of reduced cognitive testing scores [30]. Here we hypothesize that cadmium exposure could be associated with reduced cerebral blood flow in exposed ME/CFS patients because of the disruptive effects of cadmium on angiogenesis.

In order to test the hypothesis that cadmium exposure is associated with reduction in cerebral blood flow in the cortex of ME/ CFS patients, we propose to measure cadmium exposure in a cohort of ME/CFS patients compared with matched healthy controls, and then measure cerebral cortex blood flow in un-exposed healthy controls, exposed non-ME/CFS subjects, un-exposed ME/ CFS patients and exposed ME/CFS patients. Cerebral cortex blood flow could be measured with functional magnetic resonance imaging as in [30] and/or also with the transcranial sonography technique described above. In fact, with the type of setting described and using Doppler technique, we could also observe arterial vascularisation of the meninges and of cortex of the temporal lobe and pulsating arteries of about one $\mathrm{mm}$ diameter could be easily visualized (Fig. 2). The use of transcranial sonography of the temporal lobe could prove easier and certainly less expensive than functional magnetic resonance imaging and thus become part of routine examination of ME/CFS patients. Since reduction of cerebral blood flow in ME/CFS patients occurs across nearly every region assessed by functional magnetic resonance imaging [30] or by xenon-computed tomography [31], the temporal lobe could represent an easily accessible region of the human brain to be studied by transcranial sonography.

\section{Consequences of the hypothesis and discussion}

If our hypothesis is demonstrated correct, the consequences could affect prevention, early diagnosis, and treatment of ME/CFS. In addition, if an association between cadmium exposure and ME/ CFS is demonstrated, in all or in specific subsets of ME/CFS patients, this would have important repercussions in the definition of clinical cases and in forensic and insurance-related matters. Thus, in those patients, ME/CFS could be considered as consequence of occupational or accidental exposure to cadmium.

Implications in prevention are quite obvious even though there already is general consensus in limiting the exposure to cadmium because of its known carcinogenic effects. Nevertheless, demonstration of an association between cadmium exposure and ME/CFS could lead to more stringent limits for exposure as well as to the search for other symptoms in cadmium-exposed subjects in addition to those already established. Implications in early diagnosis could entail the evaluation of symptoms typical of ME/CFS in cadmium-exposed subjects and also the search for signs of exposure to cadmium in subjects diagnosed with ME/CFS. In addition, if the neurological alterations previously described in ME/CFS patients [16] could be easily and reproducibly assessed through the transcranial sonography procedure described in this study, this simple and non-expensive technique could be used in early diagnosis and follow-up of the syndrome, whether associated with cadmium exposure or not. Finally, if an association between cadmium exposure and ME/CFS is demonstrated, also the management of the syndrome could be affected. Thus, nutritional supplementation of magnesium and zinc have been proposed in the prophylaxis and therapy of cadmium exposure [32], and it is interesting to notice that an old randomised controlled trial comparing a magnesium supplement with placebo found beneficial effects of magnesium on ME/CFS patients' symptom profiles [33].
In conclusion, the hypothesis that cadmium is associated with, or even responsible for many, if not all, the symptoms and the neurological alterations typical of ME/CFS, is easily "falsifiable" according to Popper's criteria [34], and the critical tests that could prove our hypothesis wrong can be easily performed.

\section{Conflict of interest statement}

All Authors disclose any financial and personal relationships with other people or organisations that could inappropriately influence the work. The Authors declare no conflict of interest.

\section{Acknowledgements}

This study was supported by Progetto di Interesse Nazionale PRIN 2009 (M.R. and S.P.) from the Ministry of the University and Research.

\section{References}

[1] Brkić S, Tomić S, Ruzić M, Marić D. Chronic fatigue syndrome. Srp Arh Celok Lek 2011;139(3-4):256-61.

[2] Carruther BM, van de Sande MI, De Meirleir KL, et al. Myalgic encephalomyelitis: International Consensus Criteria. J Intern Med 2011; 270(4):327-38.

[3] Dinos S, Khoshaba B, Ashby D, et al. A systematic review of chronic fatigue, its syndromes and ethnicity: prevalence, severity, co-morbidity and coping. Int J Epidemiol 2009;38(6):1554-70.

[4] Holgate ST, Komaroff AL, Mangan D, Wessely S. Chronic fatigue syndrome: understanding a complex illness. Nat Rev Neurosci 2011;12(9):539-44.

[5] Lombardi VC, Ruscetti FW, Das Gupta J, et al. Detection of an infectious retrovirus, $\mathrm{XMRV}$, in blood cells of patients with chronic fatigue syndrome. Science 2009;326(5952):585-9.

[6] Groom HC, Bishop KN. The tale of xenotropic murine leukemia virus-related virus. J Gen Virol 2012;93(5):915-24.

[7] Hughlings Jackson J. Evolution and dissolution of the nervous system. In: Taylor J, editor. Selected Writings of John Hughlings Jackson, vol. 2, New York: Basic Books; 1884. p. 3-118.

[8] Roofe PG, Matzke HA. Introduction to the study of evolution: its relationship to neuropathology In: Minkler J, editor. Pathology of the nervous system, vol. 1. New York: Blakiston; 1968, p. 14-22.

[9] Sarnat HB, Netsky MG. Evolution of the nervous system. Oxford: Oxford University Press; 1981.

[10] Rapoport SI. Brain evolution and Alzheimer's disease. Rev Neurol 1988;144(2):79-90.

[11] Rapoport SI. Hypothesis: Alzheimer's disease is a phylogenic disease. Med Hypotheses 1989;29(3):147-50.

[12] Rapoport SI. Integrated phylogeny of the primate brain, with special references to humans and their diseases. Brain Res Brain Res Rev 1990;15(3):267-94.

[13] Stejskal VD, Danersund A, Lindvall A, et al. Metal-specific lymphocytes: biomarkers of sensitivity in man. Neuro Endocrinol Lett 1999;20(5):289-98.

[14] Miller R. Thimerosal, micromercurialism and chronic fatigue syndrome. Med Hypotheses 2005;64(5):1063-4.

[15] Valentine-Thon E, Müller K, Guzzi G, Kreisel S, Ohnsorge P, Sandkamp M. LTTMELISA is clinically relevant for detecting and monitoring metal sensitivity. Neuro Endocrinol Lett 2006;27(1):17-24.

[16] de Lange FP, Kalkman JS, Bleijenberg G, Hagoort P, van der Meer JW, Toni I. Gray matter volume reduction in the chronic fatigue syndrome. Neuroimage 2005;26(3):777-81.

[17] López E, Arce C, Oset-Gasque MJ, Caňadas S, González MP. Cadmium induces reactive oxygen species generation and lipid peroxidation in cortical neurons in culture. Free Radic Biol Med 2006;40(6):940-51.

[18] Hart RP, Rose CS, Hamer RM. Neuropsychological effects of occupational exposure to cadmium. J Clin Exp Neuropsychol 1989;11(6):933-43.

[19] Antonio MT, Corredor L, Leret ML. Study of the activity of several enzymes like markers of the neurotoxicity induced by perinatal exposure to lead and/or cadmium. Toxicol Lett 2003;143(3):331-40.

[20] Joyce E, Blumenthal S, Wessely S. Memory, attention, and executive function in chronic fatigue syndrome. J Neurol Neurosurg Psychiatry 1996;60(5):495-503.

[21] Haig-Ferguson A, Tucker P, Eaton N, Hunt L, Crawley E. Memory and attention problems in children with chronic fatigue syndrome or myalgic encephalopathy. Arch Dis Child 2009;94(10):757-62.

[22] Gulisano M, Pacini S, Punzi T, et al. Cadmium modulates proliferation and differentiation of human neuroblasts. J Neurosci Res 2009;87(1):228-37.

[23] Sarchielli E, Pacini S, Morucci G, et al. Cadmium induces alterations in the human spinal cord morphogenesis. Biometals 2012;25(1):63-74.

[24] Serdar MA, Akin BS, Razi C, et al. The correlation between smoking status of family members and concentrations of toxic trace elements in the hair of children. Biol Trace Elem Res 2012;148(1):11-7. 
[25] Bassi N, Amital D, Amital H, Doria A, Shoenfeld Y. Chronic fatigue syndrome: characteristics and possible causes for its pathogenesis. Isr Med Assoc J 2008;10(1):79-82.

[26] von Economo C. Temporal lobe. In: Triarhou LC, editor. Cellular structure of the human cerebral cortex. Basel; 2009. p. 114-132.

[27] Molnár Z. Evolution of cerebral cortical development. Brain Behav Evol 2011;78(1):94-107.

[28] Pacini S, Punzi T, Morucci G, Gulisano M, Ruggiero M. A paradox of cadmium: a carcinogen that impairs the capability of human breast cancer cells to induce angiogenesis. J Environ Pathol Toxicol Oncol 2009;28(1):85-8.

[29] Kim J, Lim W, Ko Y, et al. The effects of cadmium on VEGF-mediated angiogenesis in HUVECs. J Appl Toxicol 2012;32(5):342-9.
[30] Biswal B, Kunwar P, Natelson BH. Cerebral blood flow is reduced in chronic fatigue syndrome as assessed by arterial spin labeling. J Neurol Sci 2011;301(1-2):9-11.

[31] Yoshiuchi K, Farkas J, Natelson BH. Patients with chronic fayigue syndrome have reduced absolute cortical blood flow. Clin Physiol Funct Imaging 2006;26(2):83-6.

[32] Matović V, Buha A, Bulat Z, Dukić-Ćosić D. Cadmium toxicity revisited: focus on oxidative stress induction and interactions with zinc and magnesium. Arh Hig Rada Toksikol 2011;62(1):65-76.

[33] Cox IM, Campbell MJ, Dowson D. Red blood cell magnesium and chronic fatigue syndrome. Lancet 1991;337(8744):757-60.

[34] Popper KR. The logic of scientific discovery. London: Hutchinson; 1959. 